\title{
Physical fitness and autonomic dysbalance in schizophrenia
}

\author{
Karl-Jürgen Bär, Marco Herbsleb, Steffen Schulz, Tobias Rachow, Daniela Eisentraeger, \\ Christian Puta, Holger Gabriel, Andreas Voss
}

Maintaining and improving fitness are associated with a lower risk of premature death from cardiovascular disease. Patients with schizophrenia are known to exercise less and have poorer health behaviors than average. Physical fitness and physiological regulation during exercise tasks have not been investigated to date among patients with schizophrenia.

We studied autonomic modulation in a stepwise exhaustion protocol in 23 patients with schizophrenia and in matched controls, using spirometry and lactate diagnostics. Parameters of physical capacity were determined at the aerobic, anaerobic and vagal thresholds (VT), as well as for peak output. VT was correlated with psychopathology, as assessed by the PANSS, with the inflammatory markers IL-1ß, IL-6 and TNF $\alpha$ and with peak output.

The MANOVA for heart and breathing rates, as well as for vagal modulation and complexity behavior of heart rate, indicated a profound lack of vagal modulation at all intensity levels, even after the covariate carbon monoxide concentration was introduced as a measure of smoking behavior. Significantly decreased physical capacity was demonstrated at the aerobic, anaerobic and vagal thresholds in patients. After the exercise task, reduced vagal modulation in patients correlated negatively with positive symptoms and with levels of IL-6 and TNF $\alpha$. This study shows decreased physical capacity in patients with schizophrenia. Upcoming intervention studies need to take into account the autonomic imbalance, which might predispose patients to arrhythmias during exercise. Results of inflammatory parameters are suggestive of a reduced activity of the anti-inflammatory cholinergic pathway in patients, leading to a pro-inflammatory state. 